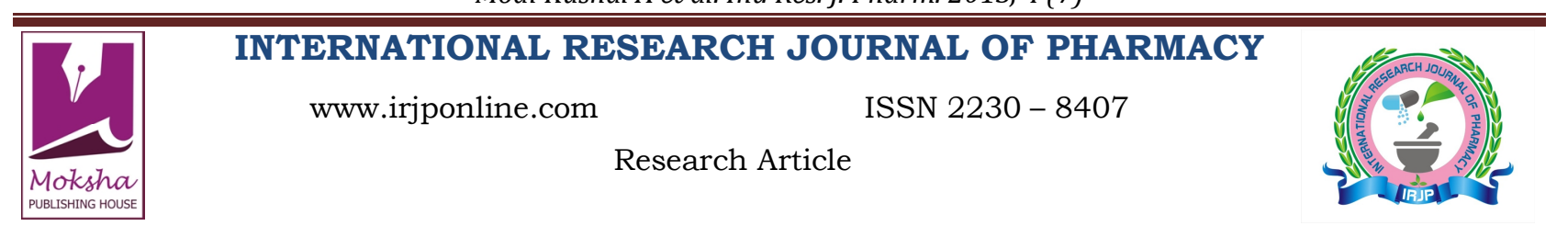

\title{
OPTIMIZATION AND EVALUATION OF NANO-LIPOSOMES OF BCS CLASS III DRUG INTENDED FOR OCULAR HERPES TREATMENT USING ARTIFICIAL NEURAL NETWORK
}

Modi Kushal A.*, Shelat Pragna K.

Department of Pharmaceutics and Pharmaceutical Technology, K. B. Institute of Pharmaceutical Education and Research,

Kadi Sarva Vishwavidyalaya, Gandhinagar, Gujarat, India

*Corresponding Author Email: kushal.modi@gmail.com

Article Received on: 23/05/13 Revised on: 09/06/13 Approved for publication: 19/07/13

DOI: 10.7897/2230-8407.04734

IRJP is an official publication of Moksha Publishing House. Website: www.mokshaph.com

(C) All rights reserved.

\section{ABSTRACT}

The objective of present experimental work was to optimize processing parameters of ocular liposomes using artificial neural network (ANN) as an optimization tool. The human eye is easily accessible site for topical dosing of drugs intended for local action or in the interior of the eye. Ganciclovir (GCV) is a synthetic analogue of 2'-deoxy-guanosine used in treatment and prevention of cytomegalovirus infections and in treatment of ocular herpes infection (orphan disease). The poor bioavailability (5-9\%) of this drug has lead development of ocular liposome to improve the bioavailability and enhance therapeutic effectiveness. GCV loaded liposomes were prepared by classical rotary flask evaporation method using HSPC/DSPG as lipid part and cholesterol as vesicle stabilizer. Box-Behnken design was applied on the selected processing parameters i.e. flask speed (X1), hydration volume (X2) and hydration temperature (X3) whereas the formulation variables were kept constant. The feed forward back propagation neural network (BPNN) method was optimized using experimental data and validated for accurate prediction of dependent variables. The obtained root mean square value of the trained ANN model was 0.00004 , which indicated that the optimal model was reached. The predicted data from ANN and the experimental data were compared by chi square test which showed no significant difference. The results conclude that the ANN model provides accurate prediction and should be further explored in optimization of novel pharmaceutical formulations.

Keywords: Ganciclovir; Liposomes; Artificial neural network; Optimization; Rotary flask evaporation method.

\section{INTRODUCTION}

The human eye is easily accessible site for topical dosing of drugs intended for local action or in the interior of the eye. The eye has its own structural benefits which allow retaining the drug in ocular sac and hence serving as a depot system ${ }^{1-3}$. The ocular route also permits non-invasive drug delivery for local delivery as well as interior delivery of drugs. Some parts of eye are almost inaccessible by systemic blood circulation and hence the disease related to these parts are ought to be treated by topical drug delivery system only. The presence and maintenance of therapeutic concentration maintenance of required drug concentration at the site of action for a desired period of time is the ultimate goal of any ideal drug delivery system. In ocular treatment the site of action may be any ocular tissue, depending upon type of disorder. Poor ocular bioavailability of drugs from the conventional dosage forms is mainly due to the irregular precorneal losses like tear production, non-productive absorption, transient residence time and the relative impermeability of corneal epithelium membrane. Due to these anatomical and physiological limitations only a small fraction of the drug (Approximately $1 \%$ or even less of the instilled dose) is ocularly absorbed ${ }^{3-5}$. The optimization of process parameters has become very necessary to achieve consistent reproducible batches. The optimization of processing steps reveals the actual relationship between process parameters and individual pharmaceutical responses. Optimization has become extremely important for dispersed delivery system like liposomes, niosomes and ethosomes, since many often interdependent factors can affect the percent drug entrapment (PDE) and vesicle mean geometric diameter. Thus, PDE and geometric mean diameter are considered as the response parameters for the study ${ }^{6}$.

In present study, we have focused on optimization of processing parameters affecting GCV loaded liposomes using the back-propagation neural network (BPNN) method. The
GCV is very poorly bioavailable (5-9\%) orally, hence the ocular herpes requires long term therapy by oral route. The liposomal drug delivery systems of various drugs are under development for ocular drug delivery purpose due to their benefits like targeted drug delivery and improved bioavailability. The liposomes were prepared by classical rotary flask evaporation method using HSPC/DSPG as lipid part and cholesterol as a vesicle stabilizer. The formulation parameters were kept constant throughout all the experiments to determine specific actions of each processing parameter. The optimization technique involves the application of BoxBehnken design to reduce the number of runs as it is more cost effective and more reliable. The selected independent variables are flask speed (X1), hydration volume (X2) and hydration temperature (X3) whereas the percentage drug entrapment (PDE) was considered as dependent variable (Y1). ANN was recognized as a potential tool for optimization. The optimized batch was further characterized by Transmission electron microscopy (TEM).

\section{MATERIALS AND METHODS \\ Materials}

Ganciclovir (GCV) was received as gift sample from Ranbaxy (super specialty) Pvt. Ltd., India. Hydrogenated soya phosphatidyl choline (HSPC) and Distearoyl phosphatidyl glycerol (DSPG) were obtained from Lipoid GMBH, Germany. Cholesterol (Chol) purchased from Sigma Aldrich, India. All other chemicals and solvents used were of analytical reagent grade and were used without further purification.

\section{Design of Experiments}

In the present study, flask speed (X1), hydration volume (X2) and hydration temperature (X3) were selected as independent variables based on single factor experimental work. Therefore, a 3 factor, 3 levels Box-Behnken design was used 
to formulate factor combination using a commercial windows based statistical software Design-Expert ${ }^{\circledR}$ version 8.0.4 (statEase, Inc., Minneapolis, MN, USA). The reduced model of factorial design helps to get results faster and more accurate. The details of experiment runs are summarized in Table 1 and 2.

\section{Artificial Neural Network (ANN)}

Commercial windows software, NeuroSolutions for Excel (NeuroSolutions) was used in the present study. ANN is a learning system based on a computational technique, which attempts to simulate the neurological processing ability of the brain $^{6-8}$. Briefly, the general structure of the ANN has three input layers, one hidden layer and one input layer. Each layer has few units corresponding to neurons. The units in the neighboring layers are fully interconnected with links corresponding to synapses. The strength of connections between two units is referred to as weights. In the hidden layer and output layer the processing unit sums its input from the previous layer and then applies the sigmoidal function to complete its output to the following layer. The input data obtained from Box-Behnken design and the results obtained of PDE (from experiments) were used to train and test ANN. Back propagation neural network system (BPNN) was used to optimize the formulation. ANN can be used to determine a non-linear relationship between independent factors and pharmaceutical responses by providing training data obtained from a designed experiment. In recent years, many pharmaceutical scientists have started to use the ANN technology in due to its précised result producing capacity and minimal errors in prediction ${ }^{8-11}$. ANN can assist formulation scientist to establish accurate dissolution profiles, process parameter optimization, various pharmacokinetic-pharmacodynamic parameters, in vitro-in vivo correlations (IVIVC) and product development. The $\mathrm{R}^{2}$ values were determined to evaluate the variability in responses. $\mathrm{R}^{2}$ value close to 1 denotes the perfect fit whereas $\mathrm{R}^{2}$ value close to 0 denotes there is no fit. The correlation coefficients (r) for each response were also determined to predict the relationship between actual and predicted outputs. The $r$ value exists in range from -1 to +1 . The $r$ value closer to +1 indicates strong positive linear relationship; $r$ value closer to -1 indicates strong negative relationship whereas the value near to zero indicates there is no such relationship exists.

ANN learns an approximate nonlinear relationship by a procedure called training, which involves varying weight values. Training is defined as a search process for the optimized set of weight values, which can minimize the squared error between the simulation and experimental data of units in the output layer.

\section{Model Training, Validation and Optimization}

A multi-layer feed forward back-propagation network that was created by generalizing the Level berg-Marquardt's learning rule to multiple layer networks and nonlinear differential transfer function was used to predict PDE of the liposomal formulations ${ }^{12-16}$. The back-propagation (BPNN) learning algorithm is the most widely used training algorithm in multi-layered feed forward networks. The network architecture consists of an input layer with three neurons, an output layer with one neuron and two hidden layer (Figure 2). The network parameters (weights) are initially set to ransom values. During the training phase, the actual output of the network is compared with the desired output and the error propagated back toward the input of the network. A neural network has two phases, commonly referred as to as the training phase and prediction phase. During the training phase, sample data containing both inputs and the desired outputs are processed to optimize the network's output meaning to minimize the deviation. The number of hidden nodes in a network is critical to network performance. Too few nodes can lead to under fitting. Too many nodes can lead the system towards memorizing the patterns in the data ${ }^{17-19}$. According to Kolmogorov's theorem, it is understood that twice the number of inputs nodes plus one is sufficient to compute any arbitrary continuous function. Input vectors and the output vector (response) were used to train the network until it can approximate a function, associate input vectors with specific output vectors. Initially one hidden layer was used and the number of hidden layer nodes was varied from $1-11$, using 100 iterations. In order to select the optimum ANN model, regression plots were constructed for the observed $\mathrm{v} / \mathrm{s}$ predicted PDE for the all test formulations. The ANN model that yielded a regression plot with a slope and $\mathrm{R}^{2}$ that were both closest to one was selected as the optimum ANN model. In order to validate the ANN model, the model was trained and check points were cross-validated using 15 formulations. Once the ANN model was trained, the model predicted the PDE for 3 checkpoint formulations. A regression plot was then constructed for the predicted PDE and observed PDE to obtain a slope and $\mathrm{R}^{2}$ to 1 as shown in Figure 4 . The determination of the final optimized model was based on the slope and $\mathrm{R}^{2}$ values.

\section{Preparation of Liposomes}

GCV liposomes were prepared by classical rotary flask evaporation method. The drug: lipid: cholesterol ratio used was 1:5:0.5 \%w/w and kept constant throughout all the experiments. The lipid mixture (HSPC/DSPG in ratio 1:3 $\% \mathrm{w} / \mathrm{w})$ and cholesterol were dissolved in chloroform: methanol $(1: 3 \% \mathrm{w} / \mathrm{w})$ mixture in rotary flask (Buchi). The solvent system was evaporated under vacuum conditions (400 $\mathrm{mmHg}$ ) at $45-50{ }^{\circ} \mathrm{C}$ temperature to obtain a thin film on the wall of rotary flask. Ganciclovir was dissolved in phosphate buffer saline (PBS) $\mathrm{pH} 7.4$ and vigorous hydration of thin film was performed using vortex mixer for 10 minutes. The aqueous dispersion was forcefully passed through Nuclepore polycarbonate membrane filters (diameter 1.0 microns, Whatman Inc. New Jersey, USA) to reduce the vesicle size. The dispersion was centrifuged at 10,000 rpm for 45 minutes and the obtained pellets were re-suspended in fresh $\mathrm{PBS}^{20,21}$.

\section{Determination of Percentage Entrapment Efficiency (PDE)}

After centrifugation, the supernant layer containing free drug was collected and filtered through whatmann filter paper. The required dilution was carried out to measure the concentration of GCV by UV-Visible spectrophotometer (UV 1700, Shimadzu, Japan). The percentage entrapment of GCV is the major key response of this experiment. The higher PDE leads to better therapeutic action and reduced free drug in dispersion $^{22}$. The entrapped drug was determined by back calculation as described in equation 1 .

$\mathrm{PDE}=$

(100 - \% amount of unentrapped drug present in supernant layer) ...... (1) 


\section{Transmission Electron Microscopy (TEM)}

The liposome vesicles were stained using Uranyl acetate and examined under 8500X magnification using Transmission electron microscope (Philips, Cm-10). The vesicles were examined for their integrity and wall firmness. The thick walled vesicles are generally less prone to degradation and leakiness. The actual shape of the vesicle can also be determined by TEM method.

\section{Chi Square Test}

Chi-square is a statistical test commonly used to compare observed data with data we would expect to obtain according to a specific hypothesis. The chi-square test is always testing the null hypothesis, which states that there is no significant difference between the expected and observed result. The chisquare is the sum of the squared difference between observed (O) and the expected (E), divided by the expected data in all possible categories and is represented in equation $2^{21}$. The chi square test was carried out to compare the predicted data for Y1 by ANN approach and actual experimental data of response Y1.

$$
\mathrm{X}^{2}=(\mathrm{O}-\mathrm{E})^{2} / \mathrm{E}
$$

Table 1: Box-Behnken Design: Casual Factors and Responses

\begin{tabular}{|c|c|c|c|c|}
\hline Runs & $\mathbf{X 1}$ & $\mathbf{X 2}$ & $\mathbf{X 3}$ & $\mathbf{Y 1}$ \\
\hline $\mathbf{1}$ & -1 & -1 & 0 & 29.85 \\
\hline $\mathbf{2}$ & -1 & +1 & 0 & 48.71 \\
\hline $\mathbf{3}$ & +1 & -1 & 0 & 31.65 \\
\hline $\mathbf{4}$ & +1 & +1 & 0 & 51.29 \\
\hline $\mathbf{5}$ & -1 & 0 & -1 & 32.66 \\
\hline $\mathbf{6}$ & -1 & 0 & +1 & 58.98 \\
\hline $\mathbf{7}$ & +1 & 0 & -1 & 34.85 \\
\hline $\mathbf{8}$ & +1 & 0 & +1 & 60.55 \\
\hline $\mathbf{9}$ & 0 & -1 & -1 & 23.55 \\
\hline $\mathbf{1 0}$ & 0 & -1 & +1 & 44.67 \\
\hline $\mathbf{1 1}$ & 0 & +1 & -1 & 40.14 \\
\hline $\mathbf{1 2}$ & 0 & +1 & +1 & 60.24 \\
\hline $\mathbf{1 3}$ & 0 & 0 & 0 & 62.31 \\
\hline $\mathbf{1 4}$ & 0 & 0 & 0 & 61.22 \\
\hline $\mathbf{1 5}$ & 0 & 0 & 0 & 61.87 \\
\hline
\end{tabular}

Table 2: Box-Behnken Design: Casual Factors and Levels

\begin{tabular}{|c|c|c|c|}
\hline \multicolumn{4}{|c|}{ Coded values of parameters of GCV liposomes } \\
\hline Levels/Factors & X1 (Flask RPM) & X2 (ml) (Hydration volume) & X3 ( $\left.{ }^{\circ} \mathbf{C}\right)$ (Hydration temperature) \\
\hline-1 & 50 & 10 & 35 \\
\hline $\mathbf{0}$ & 75 & 30 & 45 \\
\hline+1 & 100 & 50 & 55 \\
\hline
\end{tabular}

Table 3: Model Training and Testing Parameters

\begin{tabular}{|c|c|}
\hline Model training parameters & Values \\
\hline Number of hidden layers & 2 \\
\hline Number of nodes in first hidden layer & 5 \\
\hline Number of nodes in second hidden layer & 8 \\
\hline Epoch & 10000 \\
\hline Minimum mean squared error & 0.0000432334 \\
\hline Final mean squared error & 0.0000432334 \\
\hline Model testing parameters & Values \\
\hline Mean squared error (MSE) & 0.385 \\
\hline Normalized mean squared error (NMSE) & 0.051 \\
\hline Minimum absolute error & 0.081 \\
\hline Maximum absolute error & 5.978 \\
\hline
\end{tabular}

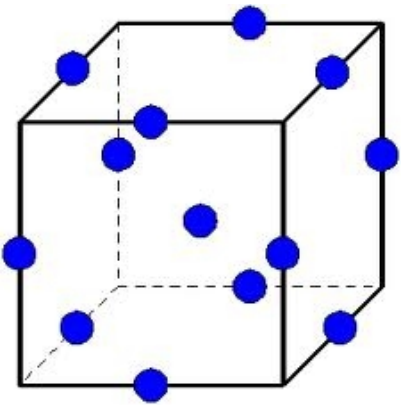

Figure 1: A Box-Behnken Design for Three Factors 


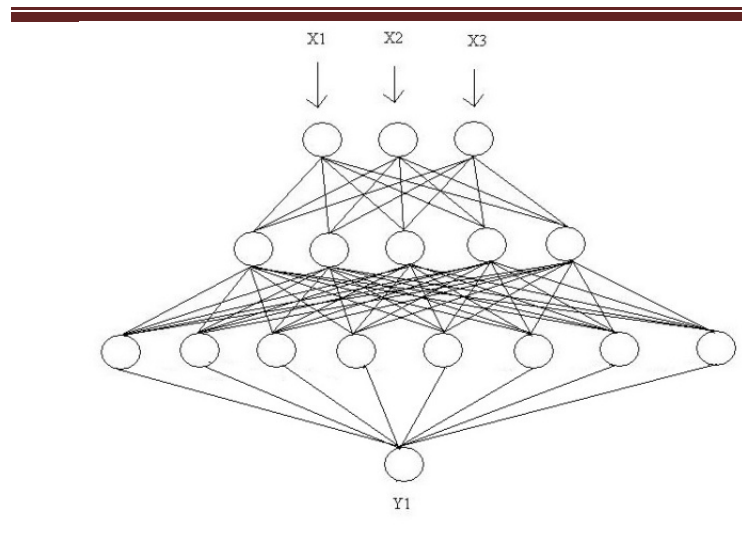

Figure 2: Structure of Proposed Artificial Neural Network

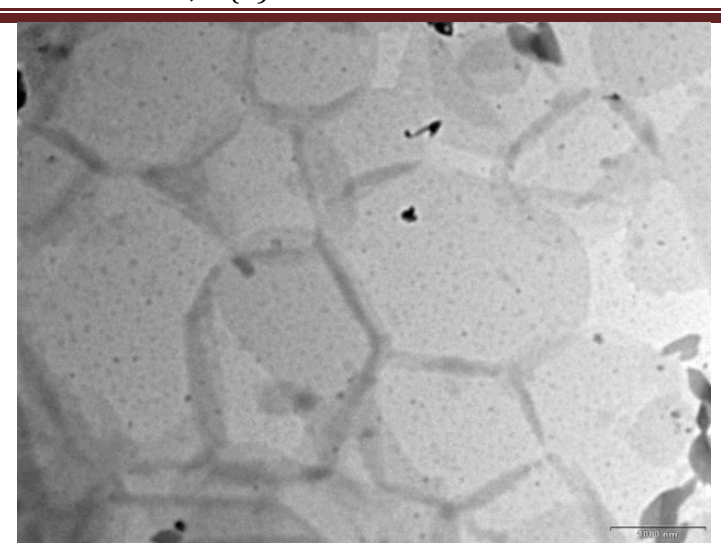

Figure 3: TEM Image of GCV Nano-liposomes

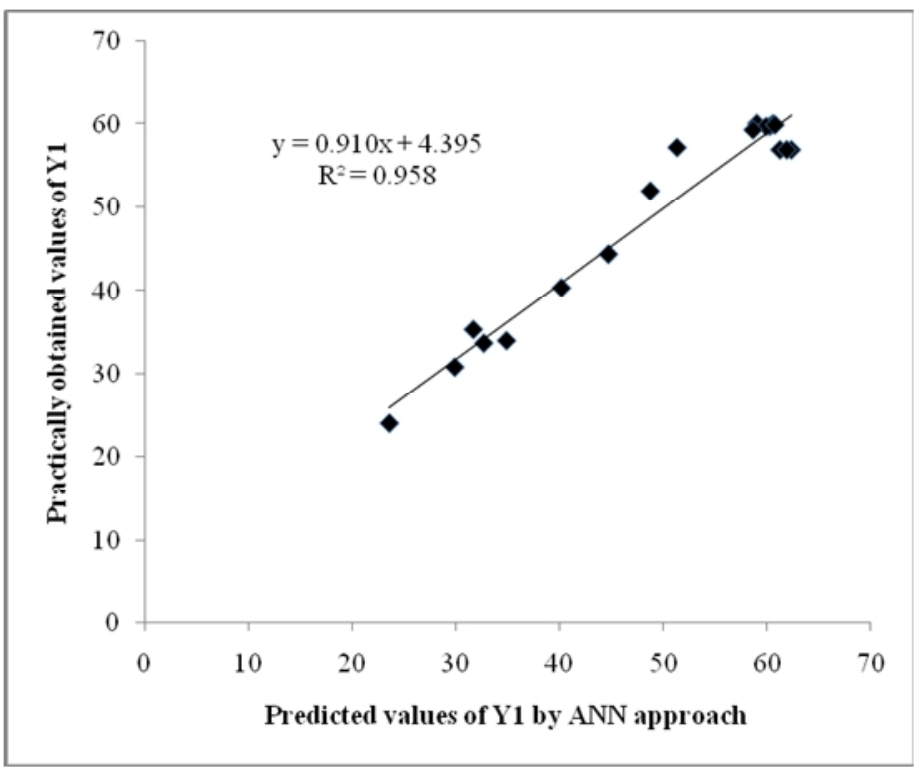

Figure 4: Graphical Comparison of Experimental and Predicted Values by ANN Model of Y1

\section{RESULTS AND DISCUSSION}

Table 2 elaborates the set data of causal factors and experimentally obtained responses (Y1). The wide rage of variation in responses indicates the critical effects of each casual factor on both the responses. It is visible from the table that PDE is more affected by the factor X2 (hydration volume) and X3 (hydration temperature). The transmission electron microscopy reveals that vesicles have thick walls hence they are less likely to leak overtime. The shape of vesicle is hexagonal, round and polygonal (Figure 3 ). The further control and fine tuning in process parameters can improve the shape uniformity of vesicles. Three independent factors; factor flask RPM (X1), hydration volume (X2) and hydration temperature (X3) were used as input of each node in BPNN method. The response obtained, i.e., PDE (Y1) was used as output layer. A set of input and output data was fed in computer and used as learning data. Modeling parameters were selected on the basis of trials and errors to obtain least mean square error (RMS). A 3-5-8-1 BPNN system was finalized for modeling and optimization of GCV loaded liposomal vesicles. It was observed that by increasing number of nodes in hidden layer led to decrease in RMS. The number of nodes showing minimum RMS was selected. Models were evaluated by average training error, minimum average test error, coefficient of determination $\left(\mathrm{R}^{2}\right)$ and correlation coefficient (r). A sharp decrease in test error was observed when it reached the optimum number of epochs during model optimization trials. The proposed ANN model was best fit as the "r" value was 0.9955 for the response Y1. The Figure 4 indicates the closeness of experimental and predicted responses on a straight line. These values were further compared by performing chi square test. The chi square value was found 2.59, which is significantly lower than the tabulated value (27.59) obtained from the chi square test table. This proves that the null hypothesis is correct and there is not significant difference between the predicted values of Y1 by ANN approach and the experimentally determined values of Y1. Thus it can be stated that ANN model predictions are reliable for PDE values of GCV nanoliposomes. Moreover; the Figure 4 represents the graphical comparison of predicted values of Y1 by ANN approach vs. practically determined values of $\mathrm{Y} 1$. The $\mathrm{R}^{2}$ value is 0.9582 which represents good linearity between both the data.

\section{CONCLUSION}

From the above results and discussion, it can be concluded that the ANN approach is accurate in predicting the output data if the model is well trained and validated. Formulation scientist can obtain précised prediction using ANN approach. There are many scopes for model building and training in 
ANN approach as compared to conventional prediction equations. Thus, the ANN approach should be further explored and applied in formulation science to get précised predictions and to reduce the experimental trials.

\section{REFERENCES}

1. Metz A. The Anatomy and Histology of the Human Eye. Nabu Press,

2. Geroski DH and Edelhauser HF. Drug delivery for posterior segment eye disease. Investigative Ophthalmology and Visual Science 2000; 40: 961-964.

3. Urtti A. Challenges and obstacles of ocular pharmacokinetics and drug delivery. Advanced Drug Delivery Reviews 2006; 58: 1131-1135. http:// dx.doi.org/10.1016/j.addr.2006.07.027 PMid:17097758

4. Mitra AK. Role of transporters in ocular drug delivery system. Pharmaceutical Research 2009; 26: 1192-1196. http://dx .doi.org/10.1007/s1 1095-009-9862-x PMid:19291373

5. Wood RW, Lee VHE, Kreuter J and Robinson JR. Ocular disposition of poly-hexyl-2 cyano[3- $\left.-{ }^{14} \mathrm{C}\right]$ acrylate nanoparticles in the albino rabbit. Int. J. Pharm 1985; 23: 175-183. http://dx.doi.org/10.1016/03785173(85)90007-9

6. Leane MM, Cumming I and Corrigan OI. The Use of Artificial Neural Networks for the Selection of the Most Appropriate Formulation and Processing Variables in Order to Predict the In Vitro Dissolution of Sustained Release Minitablets. AAPS Pharm Sci Tech 2003; 4: 215-227. http://dx.doi.org/10.1208/pt040226PMid:12916908 PMCid:PMC 2750588

7. Subramanian N, Yajnik A and Murthy RSR. Artificial neural network as an alternative to multiple regression analysis in optimizing formulation parameters of cytarabine liposomes. AAPS Pharm Sci Tech 2004; 5: 1 9.http://dx.doi.org/10.1208/pt050104

8. Takayama K, Morva A, Fujikawa M, Hattori Y, Obata Y, Nagai T. Formula optimization of theophylline controlled-release tablet based on artificial neural networks. J Control Release 2000; 68: 175-186. http:// dx.doi.org/10.1016/S0168-3659(00)00248-0

9. Takahara J, Takayama K, Nagai T. Multi-objective simultaneous optimization technique based on an artificial neural network in sustained release formulations. J Control Release 1997; 49: 11-20. http://dx.doi. org/10.1016/S0168-3659(97)00030-8

10. Takayama K, Fujikawa M, Nagai T. Artificial neural network as a novel method to optimize pharmaceutical formulations. Pharm Res 1991; 16 : 1-6. http://dx.doi.org/10.1023/A:1011986823850
11. Takahara J, Takayama $\mathrm{K}$, Isowa $\mathrm{K}$, Nagai $\mathrm{T}$. Multi-objective simultaneous optimization based on artificial neural network in a ketoprofen hydrogel formula containing O-ethylmenthol as a percutaneous absorption enhancer. Int J Pharm 1997; 158: 203-210. http://dx.doi.org/10.1016/S0378-5173(97)00260-3

12. Haykin S. Neural Networks: A Comprehensive Foundation. $2^{\text {nd }}$ ed. Englewood Cliffs, NJ: Prentice-Hall; 1999. p. 156-254.

13. Achanta AS, Kowalski IG and Rhodes CT. Artificial neural networks: implications for pharmaceutical sciences. Drug Dev Ind Pharm 1995; 21: 119-155.

14. Baughman DR, Liu YA. Neural Networks in Bioprocessing and Chemical Engineering. Academic Press, New York, NY; 1995. http:// dx.doi.org/10.3109/03639049509048099

15. Rumelhart DE, Hinton GE and Williams RJ. Learning internal representations by error propagation, Parallel distributed processing: explorations in the microstructure of cognition. MIT Press, Cambridge, MA; 1986.

16. Werbos PJ. Generalization of Back Propagation with Application to a Recurrent Gas Market Model; 1988. p. 339-356.

17. Erb RJ. Introduction to back propagation neural network computation. Pharm. Res 1993; 10: 165-170. http://dx.doi.org/10.1023/ A:1018966222807 PMid:8456062

18. Jha BK, Thambe SS and Kulkarni BD. Estimating diffusion coefficients of a micellar system using an artificial neural network. J. Colloid Interfac. Sci 1995; 170: 392-398. http://dx.doi.org/10.1006 /jcis. 1995.1117

19. Pradeep S, Jurgen V. Comparison of neural network and multiple linear regressions as dissolution predictors. Drug Develop Indust Pharm 2003; 29: 349-355.http://dx.doi.org/10.1081/DDC-120018209 PMid:12741616

20. Azmin MN, Florence AT, Handjani Vila RM, Stuart JFB, Vanlerbergh $\mathrm{G}$ and Whittaker JS. The effect of non-ionic surfactant vesicle (niosome) entrapment on the absorption and distribution of methotrexate in mice. J. Pharmacolo 1985; 37: 237-242. http://dx.doi.org/10.1111/j.20427158.1985.tb05051.x

21. Bolton S. Pharmaceutical Statistics: Practical and Clinical Applications. $4^{\text {th }}$ ed. New York: Marcel Dekker Inc; 2004.

22. New RRC. Liposomes: a Practical Approach, $1^{\text {st }}$ ed. New York: Oxford University Press; 1990.

\section{Cite this article as:}

Modi Kushal A., Shelat Pragna K. Optimization and evaluation of nanoliposomes of BCS class III drug intended for ocular herpes treatment using artificial neural network. Int. Res. J. Pharm. 2013; 4(7):154-158 http:// dx.doi.org/10.7897/2230-8407.04734 